Instituto de Estudos Sócio-Ambientais

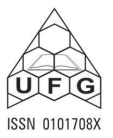

BOLETIM GOIANO DE GEOGRAFIA

v. 26, n. 1, jan./jun. 2006

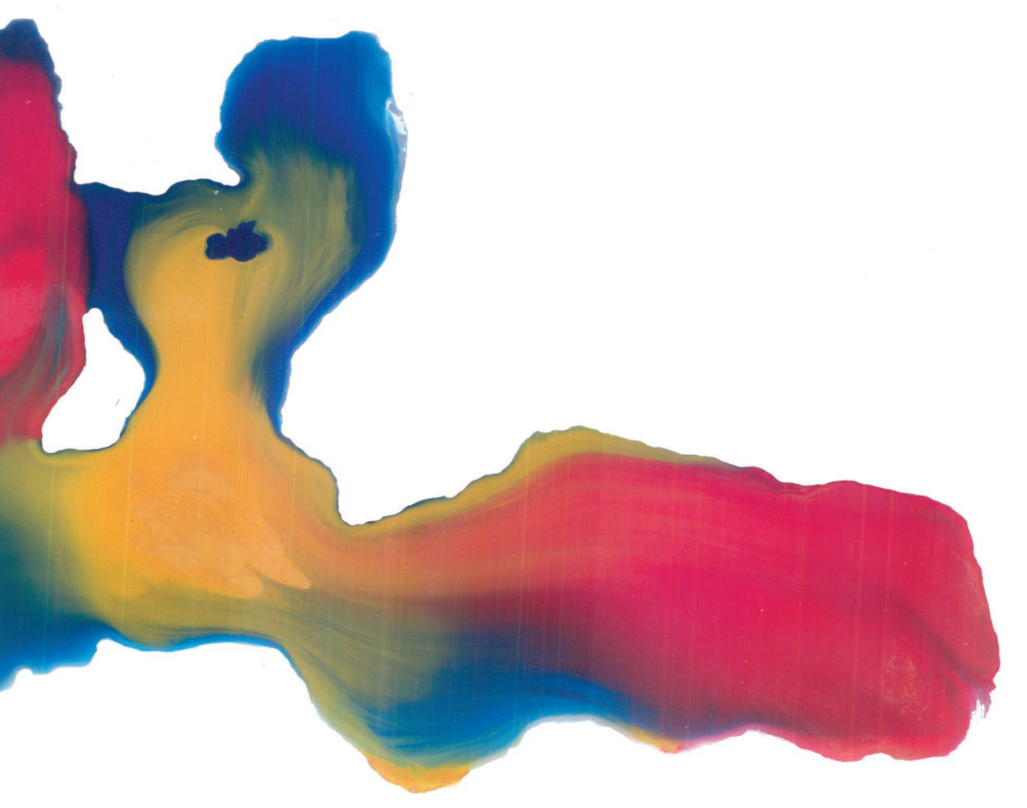

$\infty$
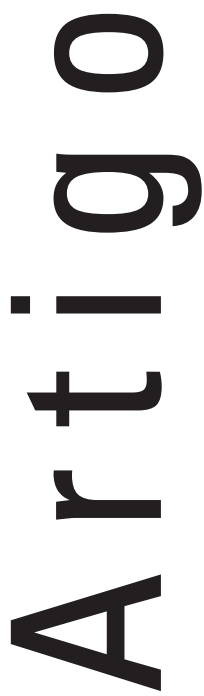


\title{
GEOGRAFÍA HUMANA Y GEOMÁTICA
}

\author{
HUMAN GEOGRAPHY AND GEOMATICS
}

\author{
Silvana Levi - Centro de Investigación en Geograía y Geomática Ing. Jorge L. Tamoyo \\ slevi@centrogeo.org.mx
}

\section{Resumen}

La geografía humana y la geomática son disciplinas que han recorrido un camino paralelo en la que a partir de la década de 1990, sus especialistas han tratado de acercarse con miras a la resolución de problemas de índole espacial. En este trabajo se plantea la relación histórica de la geomática con la geografía humana, se le enmarca teóricamente en los estudios de análisis espacial, para después ahondar en el diálogo que se ha establecido entre ambas disciplinas, y con ello responder a los cuestionamientos de iqué se ha hecho por establecer puentes entre ambas? y icómo pueden beneficiarse la una de la otra? Para responder a estas preguntas se analiza el debate que se dio en la década de los noventa entre los especialistas en sistemas de información geográfica y cartografía digital para posteriormente, a partir de finales de los 1990s, las propuestas que se hicieron desde la geografía, que con un enfoque teórico y en un intento por discutir el papel del espacio-tiempo en la geografía física y la humana, y que tocó de manera relevante la relación de la geografía con la geomática.

Palabras-claves: geografía humana, geomática, análisis espacial, visualización, cibercartografía, ciencia de la complejidad.

\begin{abstract}
For more than a decade its specialists in geomatics and human geography have discussed how put geomatics in broader frameworks which can encompass spatial social problems. This paper analyzes the history of the relationship between geomatics and human geography theoretically framed in studies of spatial analysis. It begins with the concept of geomatics, to follow with an account of the dialogue that has been established between geomatics and social sciences, especially with human geography. The paper intends to answer the following questions: how can they benefit from each other? What has been done to set a bridge between them? To answer these questions the debate which took place in the 1990's among GIS and digital cartography specialists is presented and, from 1999 the proposals, which were intended to discuss the role of space time in physical and human geography and which presents relevant approaches related to geomatics.
\end{abstract}

Key-words: human geography, geomatics, spatial analysis, visualization, cybercartography, complexity science. 


\section{Introducción}

Geógrafos en todo el mundo se acercan cada año a nuevas formas de entenderlo e interpretarlo y, en conjunto con los especialistas en geomática, traducen sus inquietudes a un nuevo lenguaje, abordan sus problemas desde nuevas perspectivas teórico-metodológicas y aprovechan las múltiples posibilidades que ofrecen hoy en día ciencias emergentes y de evolución acelerada como los sistemas de información geográfica, la cartografía digital y la percepción remota. América Latina no es ninguna excepción, tanto en la iniciativa privada, como en el gobierno y los diversos centros de investigación se desarrollan estudios en las cuales la geomática adquiere cada vez más relevancia.

Sin embargo, la relación entre geografía humana y geomática no ha sido sencilla y ha recorrido un largo trayecto con acercamientos y enfrentamientos. En este contexto se hace necesaria la reflexión acerca del camino andado, de las coincidencias y disidencias entre ambas disciplinas, de los intentos por acercarse por parte de unos y de otros, y de las posibilidades de hacer más estrecha la relación en beneficio de un mejor entendimiento del mundo que nos rodea.

Con base en lo anterior, el presente trabajo parte del concepto de geomática, para después abordar, con base en el marco teórico que ha sido el soporte de los estudios realizados en este ámbito, los diálogos que se han establecido entre dicha disciplina y la geografía humana, para analizar los intentos de acercamiento y las posibilidades que puede representar una adecuada comunicación interdisciplinaria.

\section{La geomática y su relación con la geografía humana}

La geomática o ciencia de la información geográfica es una disciplina espacial e integradora que incluye en su ámbito a la cartografía digital, los sistemas de información geográfica, la percepción remota, la fotogrametría y la geodesia. Sin embargo, la disciplina resultante va más allá que la suma de sus partes y se convierte en un sistema de pensamiento, donde no son importantes las fronteras entre las partes, ni definir el origen preciso de las aportaciones que integran su marco conceptual; sino que se conforma como una unidad orientada a dar soluciones integrales que presenta la sociedad; mismas que se abordan generalmente desde un marco teórico-metodológico 
asociado al análisis espacial, y que, si bien incorpora los campos de conocimiento mencionados, logra establecerse entre las ciencias predominantes del siglo XXI.

Geomática y geografía humana se relacionan en forma muy estrecha, ya que ambas tienen una dimensión espacio-temporal, con una historia común a partir en los años cincuentas, cuando se establecieron las bases del análisis espacial.

Los sistemas de información geográfica, la cartografía digital y la percepción remota aportan a la geografía humana posibilidades en términos de adquisición, manejo, representación y análisis de la información relevante al estudio del ser humano desde el punto de vista espacial; además de ser un instrumento de comunicación con la sociedad involucrada con el tema de estudio, lo cual puede repercutir positivamente en la investigación, gestión y en la solución de los problemas planteados.

Sin embargo, es importante recordar que durante las últimas décadas, la geografía humana desarrolló enfoques alternativos que representan una teoría crítica y una economía política más amplia para los estudios sociales. Gran parte de los autores involucrados con estas corrientes han tenido una actitud contestataria hacia la perspectiva de análisis espacial y han desarrollado sus propios discursos. Muchos de ellos han sido críticos de las limitaciones de las metodologías basados en lo cuantitativo y lo tecnológico e incluso las perciben como amenazantes, pues las asocian con una postura ideológica específica y con la reproducción del sistema capitalista; otros simplemente se han acercado a marcos conceptuales diversos, porque encuentran en ellos una mayor concordancia con sus necesidades de investigación. Un tercer grupo, el menor de los tres, se ha abocado al diálogo, a tratar de avanzar en términos teórico metodológicos para encontrarle solución a muchos aspectos que hasta la década de los ochenta, no habían podido resolverse desde el análisis espacial. A partir de lo anterior nos planteamos ihasta que punto puede contribuir la geomática a los trabajos que se desarrollan en geografía humana?, y, siendo sus marcos teórico metodológicos tan diferentes, ¿qué se ha hecho en términos de trazar un puente entre la geografía humana y la geomática?

En los años cincuenta, cuando Schaefer cuestionó la esencia misma de la geografía, los planteamientos que se hicieron en el marco de la corriente teórico metodológica que Burton llamó geografía cuantitativa, reformularon a la geografía hacia una disciplina modelada alrededor de un concepto científico particular, fundamentada en principios de objetividad y verdad, en donde se establecían 
tres dimensiones estratégicas. La primera implicaba un grado de certidumbre sobre sus observaciones empíricas y sus métodos analíticos. En este sentido, la ciencia espacial podía liberar a la geografía humana de su subjetividad. La segunda se centraba en la sistematización, y con ello, en la búsqueda del orden que subyace a las múltiples diferencias del mundo. A partir de lo anterior, se detectan patrones (forma espacial) o mecanismos de generación (proceso espacial), de tal manera que la geografía pudiera trascender las limitaciones impuestas por la descripción. La tercera integraba conclusiones en una ciencia espacial única y sistemática, a partir de los estudios de caso. Se incorpora una filosofía positivista en el ámbito de la geografía como un evento revolucionario, a partir del cual la disciplina se apropió de nuevos ideales de rigor y comprobación, lo que llevó a la construcción de una teoría más explícita. Además, la ciencia espacial introdujo un objetivo común: la organización espacial, así como una metodología común tomada del método científico, tanto para la geografía física como para la geografía humana. El desarrollo posterior y derivado de los avances del periodo positivista ha reafirmado la importancia de la teoría, aunque el enfoque teórico metodológico haya cambiado. (BARNES y GREGory, 1997, p. 1-2)

En términos tecnológicos, cabe destacar, la importancia de la aparición de las primeras computadoras en los años cincuentas y posteriormente el impulso que significó el lanzamiento de los satélites. Entonces, aún no se acuñaba el término de geomática y se hablaba de sistemas de información geográfica, cartografía automatizada y percepción remota.

Estas tres disciplinas tuvieron un auge a principios de los años ochenta, sobretodo en función de los productores de software, quienes desarrollaron herramientas muy poderosas que permitían inventariar, consultar, analizar y apoyar la toma de decisiones, ventajas que luego fueron muy difundidas por las compañías vendedoras de los mismos. En esa época, la cartografía automatizada, abrió sus posibilidades y se convirtió en cartografía digital. Con ello, ya no se limitaba a producir mapas estáticos, en los cuales la computadora solo hacía más eficientes los procesos manuales, sino que integró nuevas posibilidades más dinámicas.

En un principio, la mayoría de los proyectos relacionados con los sistemas de información geográfica utilizaron las fuentes de información existentes que se complementaron con datos obtenidos en; campo, a través de la cartografía o por medio del procesamiento digital de imágenes; con el tiempo se integró información obtenida de los sistemas de posicionamiento global GPS y fotogrametría digital.

Este proceso de masificación y la falta de conocimiento de los usuarios, hizo que se perdiera la esencia de los sistemas de información geográfica, que es el modelaje matemático para convertirse en general, en producto- 
res de mapas; esto le dio una connotación técnica que no ayudó a la imagen de la disciplina.

Lo anterior explica que todavía en 1997 se publique en Annals of the Association of American Geographers GIS tool or science?: demystifying the persistent ambiguity of GIS as "tool" versus "science" en el que se presenta un debate respecto a la polémica que todavía esta en el aire sobre si los sistemas de información geográfica son una herramienta o una ciencia (WRIGHT et al, 1997; PICKLES, 1997).

Posteriormente, los sistemas de información geográfica, tuvieron logros que fueron incorporados a la geomática en general. Entre ellos están los que permitieron modelar y buscar respuestas a cuestionamientos de tipo espacial, así como integrar datos de fuentes diversas, tales como la percepción remota, las bases de datos estadísticos y los mapas impresos. Su funcionalidad facilitó el manejo, análisis y visualización de estructuras de datos aparentemente incompatibles. Con ello cambiaron concepciones anteriores acerca de la información, su recopilación y representación; de manera tal que, por ejemplo, los mapas se convirtieron en una herramienta importante dentro de este proceso y no únicamente el producto final. La cartografía reaccionó positivamente a los cambios e integró nuevos conceptos, de manera tal que ahora hablamos de variables dinámicas, modelos digitales del terreno y modelos cartográficos digitales (KRAA, 1999, p. 157).

La cartografía digital ha cambiado radicalmente el concepto clásico del modelo de cartografía pues ha habido un rompimiento epistémico al pasar de un sistema de comunicación que representa una relación de poder entre mapas que presentan información estable y conocida y ambientes de mapeo exploratorio en los cuales se construye conocimiento. Lo anterior presenta una oportunidad para la cartografía de renovar su relación con la geografía humana crítica (CRAMPTON, 2001, p. 235-236).

\section{Geomática y ciencias sociales}

A finales de la década de los ochenta, el historiador (HARLEY, 1989) publicó un artículo, Deconstructing the map, que le dio un giro importante a la discusión sobre el acercamiento entre la cartografía y las ciencias sociales. En el artículo cuestiona la cartografía que asume que el contenido del mapa es una representación precisa y que refleja una realidad objetiva. Harley (1989) propone a los mapas como textos en cuya producción intervie- 
nen también factores culturales y políticos. A partir de la obra de (HARLEY, 1989) se amplió la discusión no sólo sobre cartografía sino también sobre los sistemas de información geográfica, para considerarlos en sus contextos institucionales, sus discursos y prácticas para propiciar debates más amplios dentro de la teoría social, sobre la triada ciencia-tecnología-sociedad, en los que, por un lado se veía la economía política de la informática y, por otro, se ponía a la cartografía dentro de los debates más amplios en geografía humana.

En 1990 hubo una polémica que se inició con un editorial de Peter Taylor en el Political Geography Quarterly (Schuurman, 1999, p. 572) en el que planteó que aunque los sistemas de información geográfica estaban equipados para manejar información, eran inadecuados para producir conocimiento. Participaron en el debate especialistas de sistemas de información geográfica, entre los que destaca (OpENSHAw, 1990) quien representa a los SIGs como tecnologías creadoras de conocimiento que pueden reintegrar la geografía. Schuurman (1999), hace un recuento muy detallado de la polémica de los años 1990 y analiza cada etapa en la discusión sobre la relación de los sistemas de información geográfica y teoría social.

En un primer momento, los especialistas en sistemas de información y los científicos sociales manejaban lenguajes diferentes por lo que la comunicación tuvo grandes tropiezos, sin embargo, esta discusión fue el inicio de un dialogo que se concretizó en una primera reunión en 1993, cuyo contenido se publicó en (SHEPPARD y POIKER, 1995).

En noviembre de 1993 hubo una iniciativa del National Center for Geographic Information and Analysis de organizar una reunión sobre las implicaciones sociales de cómo la gente, el espacio y el medio ambiente se representan en los sistemas de información geográfica. En esa reunión tuvo lugar en 1996 y en ella se plantearon tres asuntos centrales en términos conceptuales referentes a sistemas de información geográfica y sociedad, y con base en ellos se orientaron las discusiones. El primero de ellos fue la epistemología; el segundo fue sistemas de información geográfica, instituciones de datos espaciales y el acceso a la información y el tercero fue el desarrollo de los SIGs alternativos. De ahí salieron varias líneas de investigación (National Center for Geographic Information and Analysis, 1996).

A mediados de los noventa se publicaron otras dos obras importantes para el debate en cuestión. La primera intitulada Ground Truth: The social implications of Geographic Information Systems, editado por John Pickles en 1995 y la segunda fue un número de la revista Cartography and GIS (CAGIS) editada 
por Eric Sheppard y Tom Poiker, también en 1995, con los resultados de la reunión que tuvo lugar en noviembre de 1993 (SchUURman, 2000, p. 578).

Los planteamientos anteriores se pueden resumir sobre la base de que resultados de cualquier tipo de sistemas de información geográfica están relacionados con la inteligencia humana y que esta a su vez, le da significado a dichas estructuras del conocimiento. Esto implica que la forma correcta de analizar la información geográfica no es algo determinado a priori, sino que se logra en concordancia con quien la está viendo y en función del propósito para el cual fue creado. En los sistemas de información geográfica hay cuatro perspectivas interrelacionadas: empíricas, formales, derivadas de la experiencia y sociales, cada una con su punto de vista del tiempo y el espacio (Couclelis, 1999, p. 35).

Esto es particularmente importante ya que las nuevas formas de información geográfica y la cibernética desempeñan un papel primordial en la solución de problemas de la sociedad (PicKLEs 1999, p. 53). En ese sentido son interesantes los ejemplos de aplicaciones elaborados en el Centro de Investigación en Geografía y Geomática (CentroGeo) de cartografía cibernética, geo-textos y soluciones integrales de geomática.

Entre los intentos de acercamiento merecen especial atención el trabajo de (SCHUURMAn, 2002) Reconciling social constructivism and realism in GIS y el de (Mei-Po Kwan, 2002) Feminist visualization: Re-envisioning GIS as a method in feminist geographic research. Schuurman (2002) elabora un trabajo de corte teórico de una especialista en sistemas de información geográfica en busca de comunicación con las ciencias sociales. La autora resume sus planteamientos de la manera siguiente:

Los usuarios y desarrolladores de SIGs asumen que estos modelan a la realidad y por lo tanto pueden usarse para explicar y predecir procesos; esta posición realista, bajo el cual se realizan una buena parte de los trabajos en geomática, no toma en cuenta las influencias sociales en el análisis y representación de los datos. La autora trata de construir un puente entre la postura filosófica realista y la del constructivismo social. Para ello analiza tres ejemplos a) la construcción de modelos como procesos sociales, b) el desarrollo de algoritmos como metáfora social y c) los parámetros sociales en la investigación sobre generalización cartográfica. Resalta las implicaciones realistas y destaca que la tecnología es afectada por las culturas en las que se desarrollan y aplican los SIGs; considera que éstos son una adecuada representación de los fenómenos espaciales y son útiles en la predicción de eventos futuros. Pero, para considerar el contexto socio-académico donde se producen estos trabajos, afirma que se deben identificar constructivamente los parámetros sociales de la tecnolo- 
gía, a manera de lograr colaboración entre especialistas interesados en la influencia social de los SIGs y los especialistas que se interesan en su desarrollo. (SchUURMAN, 2002, p. 74)

El gran mérito del artículo publicado por Shuurman fue el hecho que aparece en una revista electrónica de geografía crítica, la revista $A C M E$, lo cual, en un esquema de comunicación implica haberse acercado y establecido un primer diálogo con geógrafos humanos.

Por su parte, Kwan (2002), explora la manera en que los sistemas de información geográfica pueden usarse para la investigación geográfica feminista. Con base en ello explora las posibilidades de los SIGs para proponer re-imaginarlos como un método, además plantea a la visualización como una práctica posible en la investigación crítica feminista.

Los SIGs tienen capacidad para desplegar y sobreponer capas de datos que pueden revelar contextos espaciales, representar conexiones espaciales y eventualmente sugerir las relaciones sociales en su complejidad; sin embargo, todavía no permiten estudiar los procesos complejos que participan en la construcción social del espacio. Lo anterior traza líneas para la investigación cualitativa. Los SIGs pueden usarse en varios sentidos, ya sea para indicar áreas que requieren atención urgente, para intervenir en el inicio de un cambio socio-político y otras. (KwAN, 2002, p. 646)

Actualmente se están desarrollando técnicas que no necesariamente se adaptan a las formas de pensar tradicionales, incluyendo la posibilidad de tomar en cuenta cada vez más el aspecto social y resolver retos tales como el tratamiento de lo local, la visualización y las nuevas implicaciones de la cartografía cibernética, entre otros, mismos que se analizarán a continuación.

\section{Local}

El tratamiento de lo local parte de la idea que la geografía es la disciplina cuya razón de ser es la explicación de la diferencia y la diversidad.

Para ello, los geógrafos requieren de perspectivas teóricas que permitan dilucidar los principales perfiles de ambas, así como esclarecer el significado de las divergencias y aclarar los patrones contradictorios de la diferenciación espacial, en un mundo cuya complejidad es cada vez mayor, con intercomunicaciones globales cada vez más estrechas, donde coexisten desigualdades extremas entre localidades. (Mc DowelL, citada por Johnston, 1999, p. 40) 
Desde los sistemas de información geográfica (KwAN, 2002, p. 649) plantea que lo local, en su relación con la investigación geográfica, ayuda a entender la experiencia de los grupos sociales a través de distintos ejes de diferencia.

En concordancia con lo anterior, existe en la actualidad, dentro de la escuela de análisis espacial, una importante tendencia que busca enfocarse en las diferencias espaciales, en oposición a los trabajos de la primera época, cuando los investigadores y profesionistas se centraban en las semejanzas. Para ello, adquieren mayor relevancia los datos locales, especialmente si en ellos se resaltan las particularidades del territorio que resultan excepcionales. Lo anterior es un avance respecto a la época en la cual la geografía se dedicaba a la búsqueda de generalizaciones y leyes, ya que reflejan de manera más cercana la realidad; además ayuda a responder ciertas preguntas sobre la sociedad actual, con un enfoque más crítico. La obtención de estadísticas que reflejen lo local hace que cada vez más, sea posible tener conjuntos de datos espaciales mayores y cada vez más complejos. (Fotheringham, 1997, p. 88)

A pesar de lo anterior, la obtención de datos locales significativos que ayuden a la explicación de fenómenos geográficos y a su interpretación teórica no es sencilla y se enfrentan a una serie de obstáculos.

La problemática inicia con la falta de información, el acceso a la misma, su confiabilidad y la complejidad metodológica para la producción, manejo y sistematización de datos que reflejen las realidades locales, en todas sus dimensiones, desde las que entran en el ámbito de lo físico concreto, hasta aquellas que se construyen en las esferas sociales, políticas y en general relacionadas con la mente humana y la forma en que sus ideas se concretan en el territorio. En este sentido, los datos cualitativos se enfrentan a mayores dificultades que los cuantitativos. Las percepciones sociales generalmente no se toman en cuenta, como si no formaran parte de la organización, construcción y dinámica de los territorios por lo que incorporar todas las esferas de lo humano, incluidas las anteriores, están siendo abordadas y ante ellas existen propuestas concretas que llevan a la explicación de problemas y fenómenos locales. (LONGLEY, 2003, p. 117)

Un ejemplo de lo anterior, en el cual se ha colaborado desde CentroGeo en México, ha sido el desarrollo, dentro del campo de la geomática, de la cartografía participativa, diseñada en un inicio para solventar la falta de información espacial, pero que ha tenido un alcance mayor pues permite incorporar el conocimiento y las vivencias de los diferentes actores involucrados en un territorio y con referencia a una problemática local específica (Luscombe y Reyes, 2004). 


\section{Visualización}

Se puede definir a la visualización como un campo de la graficación por computadora que se enfoca a los aspectos analíticos y de comunicación de la representación visual por lo que tiene que ver con la generación de nuevas formas de ver la información para crear conocimiento.

Es importante distinguir entre la visualización en la cuál el énfasis está en la exploración de los datos y la construcción del conocimiento y la de la cartografía tradicional en la cual el énfasis es la representación y difusión del conocimiento. Aunado a lo anterior, lo visual se ha abordado como un área de la disciplina que se encuentra en desarrollo y que implica un modelo de comunicación. En cartografía digital se ha redefinido el concepto de visualización pasando del concepto de simplemente "hacer visible" a la de una tecnología moderna que ofrece la oportunidad de una visualización interactiva en tiempo real. (KRAAK, 1999, p. 158)

Los mapas tienen un papel fundamental como parte metodológica y en el lenguaje del geógrafo. La investigación cartográfica se enfoca en la actualidad en nuevos temas de representación y visualización, basados en los estudios empíricos. Lo anterior permite entender mejor los procesos cognoscitivos sobre la información espacial. Asimismo, la teoría social crítica plantea re-concebir el mapa y a la visualización como una práctica social. En este sentido se relacionan la elaboración del mapa con factores socioeconómicos y el ejercicio del poder (HARLEY, 1996; KING, 1996)

El mapa, bajo estos nuevos conceptos ya no es un modelo geo-referenciado dibujado en papel, sino un conjunto de datos espaciales organizados en torno a una estructura virtual que permite una visualización dinámica, en donde se pueden reflejar muchos puntos de vista: el del investigador, del tomador de decisiones, de la población estudiada y del lector del mapa. (KRAAK, 1999, p. 158)

Por medio de la visualización, las relaciones espaciales se hacen visibles en los mapas, gráficos, imágenes a través de las cuales se representa la construcción social del conocimiento cartográfico (CRAMPTON, 2001, p. 244).

Lo anterior nos presenta un rompimiento epistémico con el modelo de cartografía concebido como un sistema de comunicación en el que los mapas presentan información estable y conocida para pasar a ambientes exploratorios en los cuales se construye el conocimiento, lo que permite construcciones sociales y representa una oportunidad para la cartografía de relacionarse más directamente con la geografía humana. (CRAMPTON, 2001, p. 235) 
La visualización geográfica está relacionada con la construcción e interpretación de imágenes (THornes, 2004, p. 787). En geografía la visualización es un medio importante para la producción del conocimiento ya que puede mostrar las diferencias entre los efectos sociales de las diferentes visiones (KWAN, 2002, p. 649).

\section{Cartografía cibernética}

Los desarrollos teóricos en la geomática apuntan ahora más que nunca a la posibilidad de ampliar su relevancia en enfoques centrados en el análisis espacial y en la construcción de puentes hacia otras formas de pensamiento geográfico. En este sentido es importante mencionar a la cartografía cibernética; en ella sobresalen los trabajos de (REYES, 2005), (REYES y MARTínez, 2005) y (MARTíNEZ y ReYES, 2005), en los que el enfoque cibernético de las dos autoras les permite sentar las bases para la construcción de marcos teóricos propios de la geomática en donde de manera explicita se entrelazan conocimientos y metodologías tradicionalmente considerados como de ciencia básica con ámbitos de soluciones a problemas planteados por distintos sectores de la sociedad. Las autoras consideran que el lenguaje propio de esta nueva ciencia, a diferencia de lenguajes anteriores de la cartografía, percepción remota y sistemas de información geográfica, brinda elementos teóricos que permitirán encontrar espacios comunes de reflexión con los distintos enfoques geográficos y que apuntan hacia un proceso de sinergia entre la geografía humana y la geomática mucho más amplio que el que hasta ahora se ha logrado.

\section{El puente desde la Geografía}

En términos generales, el análisis de la obra escrita posterior al debate entre investigadores de SIGS y sus críticos nos muestra que estas discusiones han influido en la consideración de los investigadores dedicados a la geomática de estudiar problemas políticos y sociales pero se requiere un mayor enfoque al debate teórico sobre la relación de la geomática con la geografía humana.

Sin embargo, desde la geografía, se plantean bases importantes, justamente por ser de corte teórico, para la discusión, en el artículo "Space-time, 'science' and the relationship between physical geography and human geo- 
graphy" de (MASSEY, 1999), en el que por un lado la autora reflexiona sobre la naturaleza del espacio, a partir de las diferencias teórico-metodológicas existentes entre geografía física y geografía humana, y por otro, aborda la complejidad de la geografía en el marco de las ciencias en general.

Este artículo y su posterior discusión, analizan el papel del espaciotiempo y la forma tradicional de asumir el modelo de la física y como esto afecta la relación entre geografía física y la humana. Este artículo y la discusión posterior del mismo, tiene implicaciones importantes en la relación de la geografía humana con al geomática.

Massey (1999) explora la posibilidad de que hay aspectos en común entre la geografía física y la humana en las formas emergentes de conceptualizar el espacio, el tiempo y el espacio-tiempo. Para ello retoma a dos artículos; uno de Jonathan Raper y David Livingstone, "Development of a geomorphological spatial model using object-oriented design” En este artículo los autores desarrollaron un modelo, sobre la base de conceptos de la geografía física, con el uso de computadora para el análisis de los procesos espacio temporales, mismos que están fuertemente influidos por el modelo de espacio y de tiempo usado. El artículo de David Sudgen, "The east Antartic ice sheet: instable ice or instable ideas". Éste último argumenta que la geomorfología debe entenderse como una ciencia compleja y sintética que combina el estudio de procesos temporales y atemporales y no como una física imperfecta (MASSEY, 1999, p. 266)

De acuerdo con Massey (1999, p. 262), Raper y Livingstone destacan la importancia del espacio relativo en la representación y modelaje de problemas ambientales, de tal manera que, los resultados obtenidos en los estudios de los procesos espacio-temporales, están influidos por los modelos de espacio y tiempo que se adoptan al elaborarlos.

Massey (1999, p. 263) analiza la alusión de los autores antes mencionados a los desarrollos teóricos en física, a partir de los cuales el espacio y el tiempo que son caras de la misma moneda, son conceptos relativos a la naturaleza y comportamiento de los entes que los habitan. Es decir:

El espacio y el tiempo no son marcos rígidos que existen independientemente de aquello que se desarrolla sobre ellos. A partir de lo anterior distinguen dos posibilidades de desarrollar modelos del espacio: el geométrico y el orientado a objetos. El primero sitúa a las coordenadas geográficas como la estructura de representación espacial primordial, lo cual aplican al problema analizado. El segundo define la naturaleza de las entidades que se pretende analizar en función de sus características y comportamiento para establecer la estructura de representación espacial. 
Lo anterior, plantea Massey, tiene importantes implicaciones para la geomática, pues:

plantea la disyuntiva entre la posibilidad de ubicar el fenómeno de análisis en un marco espacio temporal determinado o establecer, en función de las entidades que se pretende estudiar, un conjunto de mundos, en donde cada uno de estos tiene un sistema de referencia espacial que incluye al tiempo.

En este punto se hace énfasis que tiempo y espacio no están separados en tanto son una propiedad de los objetos, y que forman parte de un mismo sistema (MASSEY, 1999, p. 262).

En un enfoque orientado a objetos, éstos preceden al espacio-tiempo o se dan en simultaneidad. Lo anterior lo relaciona la autora con lo que plantea un grupo de geógrafos humanos en Inglaterra, en el que ella se incluye, quienes han repensado el espacio-tiempo como un todo, en cuyo concepto hay que tomar en cuenta tres aspectos: a) es relativo, es decir, está definido por las entidades que lo componen; b) es relacional en cuanto se constituye a través de la operación de las relaciones sociales que constituyen estas entidades; y c) es integral a la constitución de las mismas entidades ya que éstas son espacio-tiempo locales. (MASSEY, 1999, p. 262)

Critica la forma en que se han retomado los modelos de la física para la investigación en ciencias sociales, lo cual deriva en una concepción de lo social como un sistema simple con mucho ruido, en lugar de concebirlo como un sistema complejo (Massey, 1999, p. 263). En este sentido es esencial reconocer la complejidad que implica el análisis geográfico, ya que tanto la geografía física como la humana son ciencias complejas sobre sistemas complejos (MASSEY, 1999, p. 266).

La autora plantea que tiempo y espacio no deben separase, en función de que el segundo es la base de la multiplicidad, que conforma interacciones que producen diferencias. Con ello se producen los cambios, y el tiempo es el vehículo de dicho cambio y no la causa del mismo. Para mantener abierto el tiempo, el espacio debe imaginarse como la esfera de existencia de la multiplicidad y la diferencia. (MASSEY 1999, p. 273)

Se pregunta ¿como puede esto relacionarse para volver a conceptualizar la especialidad en otros campos de la geografía incluyendo a la geomática? y finaliza planteando un reto: "conversemos", dice.

Raper y Livingstone (2001) aceptan el reto y presentan sus convergencias y divergencias respecto al artículo de Doreen Massey en un artículo in- 
titulado "Let's get real: spatio-temporal identity and geographic entibies." En el retoman lo dicho por (MASsEy, 1999, p. 269) en el sentido de que la representación del espacio-tiempo emerge de la conceptualización de dichas entidades y proponen cuatro debates teóricos: a) el papel de la representación; b) la conceptualización de las entidades; c) el papel del espacio-tiempo; y d) la constitución mutua de las entidades espacio-temporales y el proceso de la representación (RAPER y LIVINGSTONE, 2001, p. 238).

Con respecto a la geomática plantean que, en la actualidad, hay limitantes para representar digitalmente los fenómenos ambientales y sociales complejos lo cual ha estimulado discusiones sobre al estado científico y filosófico de sus métodos. En ellos destaca la naturaleza de las ontologías y epistemologías espacio-temporales y deriva esencialmente en la experimentación y en la representación multidimensional. (RAPER y LIVINGSTONE, 2001, p. 240)

En el debate también participa (LANE, 2001) con el artículo "Constructive comments on D Massey: space-time, "science" and the relationship between physical and human geography”, donde desde la geografía física, presenta convergencias y divergencias.

Lane (2001, p. 252), reconoce la importancia de las limitantes aunque considera que no se debe priorizar a ninguna en particular

El conocimiento está condicionado por las decisiones que se toman al plantear la investigación, los lugares que se visitan durante el proceso y el conocimiento del investigador de área dentro de la cual se lleva a cabo el trabajo. Esto lleva al investigador a través de de un conjunto de ambientes complejos en una forma que parece racional a nivel individual aún cuando no lo sea para otros. La diferencia entre lugares se da no solo porque estos no son iguales pero también porque la forma en que estos son abordados por cada investigador.

Finalmente un comentario final de (MASsey, 2001): "Talking of space -time"de esta etapa de la discusión. En el diálogo entre estos autores queda claro que existen canales de comunicación importantes.

La discusión anterior se ha continuado en dos talleres del Institute of British Geographers en el 2000 y 2001 y en su reunión anual de 2003, para después seguir en la reunión de la Unión Geográfica Internacional en el 2004.

En el 2004 (O’Sullivan, 2004) a su vez, responde a la invitación de (MASSEY, 1999) a la discusión y participa en lo que considera un intercambio de ideas constructivo y analiza a la ciencia de la complejidad, sobretodo en su relación con la geografía humana. 


\section{Ciencia de la complejidad}

O’Sullivan (2004) sugiere ver a los modelos como una fuente de narrativas geográficas que puede ser útil para promover un enlace que tome en cuenta las diferentes perspectivas en la geografía (O'Sullivan, 2004, p. 282).

La complejidad está conformada por 3 categorías: complejidad algorítmica, complejidad determinística y complejidad agregada. De las anteriores, el autor considera que la tercera es la más importante para la geografía, es decir, la complejidad agregada o estudio de fenómenos caracterizados por interacciones entre componentes diferentes, en la cual la configuración espacial local afecta los resultados de todo el sistema.

En la complejidad agregada se enfoca la forma en que las relaciones entre entidades están diferentemente estructuradas a través del sistema espacial. A distintas escalas las estructuras de interacción se configuran y reconfiguran por efectos espaciales, aunque no son espaciales por si mismas. El lugar es una red de relaciones sociales, políticas, económicas y otras que están estructuradas espacialmente y configuradas en el tiempo. (O’Sullivan, 2004 p. 284)

La ciencia de la complejidad está fundamentalmente determinada por las decisiones ontológicas que toma el investigador como resultado del enfoque epistemológico que se basa en construcciones de modelos que simulan la realidad. Empieza con fenómenos complejos del mundo real, se representan los fenómenos en un modelo de cómputo. Las entidades y relaciones son planteamientos teóricos relacionados al tema de estudio de tal manera que el modelo representa una teoría sobre el mundo y no el mundo en sí. El resultado final es un modelo complejo cuyo comportamiento puede ser tan complicado como lo es la realidad que representa. (O’SulLivan, 2004, p. 291)

O’Sullivan (2004) sugiere una manera de ver la ciencia de la complejidad en la cual los modelos se entienden como una fuente de narrativas geográficas, que analizan al mundo y lo proyectan hacia el futuro. Desde esta perspectiva, considera que "es importante que las teorías representadas en los modelos se examinen y evalúen de la misma forma crítica que se hace en las ciencias sociales" (O’Sullivan, 2004, p. 291).

De la discusión anterior se deriva que para trazar puentes entre la geomática y la geografía humana se debe privilegiar la discusión sobre los temas de investigación, desde un enfoque teórico-metodológico, aceptando la diversidad y tomando en cuenta que ambas son ciencias complejas que estudian fenómenos espacio-temporales y que para ello es importante propiciar el intercambio de ideas y no institucionalizar las diferencias. (O’Sulurvan, 2004, p. 291) 


\section{Conclusiones: hacia el futuro}

Geomática y geografía son disciplinas que caminan independientes pero entrelazadas, que se encuentran y crean sinergia en distintos momentos de su desarrollo, la gran cantidad de artículos sobre el tema de geomática - incluyendo aquellas sobre SIGs y cartografía digital - en las principales revistas de geografía humana es una muestra de ello., La perspectiva social, por su parte se puede identificar como una parte relevante de la geomática. No necesariamente todo lo que se hace desde el punto de vista social, cultural, económico, histórico o político y que tiene un enfoque espacial, puede ser analizado, en la actualidad, a través de las propuestas teóricas y metodológicas que se encuentran dentro del campo de esta ciencia. A pesar de lo anterior, la posibilidad para integrar ambos marcos conceptuales son muchas más de las que se han explorado hasta la fecha, por lo que las relaciones e intersecciones entre ambas disciplinas presentan un reto y un largo camino a seguir.

Este camino señalado a partir de la posibilidad de establecer un diálogo entre la geomática y las corrientes alternativas al análisis espacial en geografía humana representa un gran reto, que abre múltiples posibilidades hacia el futuro, sin que ello quiera decir que todos los aspectos de la geografía humana puedan y/o deban ser abordados desde el análisis espacial.

Hasta ahora, la discusión con miras a crear puentes que unan ambas disciplinas ha sido amplia desde la geomática, sin embargo, han sido importantes, por su profundidad desde le punto de vista teórico-metodológico, los cuestionamientos planteados desde la geografía con la discusión iniciada por (MASSEY, 1999); pues aún cuando se refiere a la relación geografía física y la humana mucho de lo que ahí se dice tiene relevancia en el tema que estamos tratando. Desde el punto de vista de la relación de la geomática con la geografía humana me parece importante retomar la propuesta de (O’Sullivan, 2004) ya que ambas son ciencias complejas que estudian el espacio-tiempo.

De acuerdo con lo anterior y considerando que el espacio-tiempo es una estructura que subyace a los problemas de investigación planteados por la geografía humana y la geomática, el desarrollo de ambas disciplinas pueden encontrar rutas paralelas, que permitan la comunicación en beneficio de las temáticas en las que coincidan. 


\section{Referências}

BARNES, Trevor y GREGORY, Derek. Reading Human Geography. The poetics and politics of inquiry. London, New York: Arnold, 1997.

COUCLELIS, Helen. Space, time, geography. In: LONGLEY, Paul, GOODCHILD, Michael F., MAGUIRE, David J., RHIND, David W. (ed.). Geographical Information Systems. Segunda edición. New York: John Wiley \& Sons Inc, 1999.

CRAMPTON, Jeremy W. Maps as social constructions: power, communication and visualization. Progress in Human Geography, Reino Unido, 25:2, 2001.

FOTHERINGHAM, A. Stewart. Trends in quantitative methods I: stressing the local. Progress in Human Geography, Reino Unido, 1997.

HARLEY, J.Brian. Deconstructing the map. In: AGNEW, John, LIVIGSTONE, David N. y ROGERS, Alistair (ed.). Human Geography; an essential anthology. Oxford: Blackwell publishers. 1996. Reproducción del artículo publicado en Cartographica, Canadá, 26.1989.

JOHNSTON, Ronald J. Geography and GIS. In: LONGLEY, Paul, GOODCHILD, Michael F., MAGUIRE, David J., RHIND, David W. (ed.). Geographical Information Systems. Segunda edición. New York. John Wiley \& Sons, 1999.

KING, Jeoff. Mapping reality: an exploration to cultural cartographies. New York: St. Martin Press, 1996.

KRAAK Menno J., Visualizing spatial distributions. In: LONGLEY, Paul, GOODCHILD, Michael F., MAGUIRE, David J., RHIND David W. (ed.). Geographical Information Systems, segunda edición. New York: John Wiley \& Sons inc, 1999.

KWAN, Mei-Po Feminist visualization: Re-envisioning GIS as a method in feminist geographic research. Annals of the Association of American Geographers, Estados Unidos, 92:4, 2002.

KWAN, Mei-Po. GIS methods in time-geographic research: geocomputation and geovisualization of human activity patterns. Geografiska Annaler, Reino Unido, 86 B, 2004.

LANE, Stuart N. Constructive comments on D Massey: space- time, "science" and the relationship between physical and human geography. Transactions of the Institute of British Geographers, Reino Unido, 26, 2001.

LONGLEY, Paul A. Spatial analysis in the new millenium. Annals of the Association of American Geographers, Estados Unidos, 90, 1, 2000.

LONGLEY, Paul A Geographical Information Systems: will developments in urban remote sensing and GIS lead to "better" urban geography? Progress in Human Geography, Reino Unido, $26: 2,2002$.

LUSCOMBE, B. Wayne y REYES, Carmen. Building consensus in environmental decision making. A methodology integrating GIS tools and structured communication. Proceedings of the National Association of Environmental Professionals meeting, Estados Unidos, 2004.

MARTÍNEZ, Elvia y REYES, Carmen. Cyber-cartography and society. In: TAYLOR, D. R. F. (ed.) Cyber-cartography: theory and practice. Reino Unido, Elsevier Scientific, 2005

MASSEY, Doreen. Space-time, 'science' and the relationship between physical geography and human geography. Transactions of the Institute of British Geographers, Reino Unido, 24, 1999. 
MASSEY, Doreen. Talking of space-time. Transactions of the Institute of British Geographers, Reino Unido, 26: 2001.

OPENSHAW, Stan. A view on the GIS crisis in Geography. In: AGNEW, John, LIVIGSTONE, David N. y ROGERS, Alistair (ed.). Human Geograph: an essential anthology. Oxford Blackwell publishers, 1996 Reimpresión de: A view on the GIS crisis in Geography, or, using GIS to put Humpty Dumpty back together again, Environment and Planning A, Inglaterra, 23, p.621-628, 1991.

O'SULLIVAN, David. Complexity Science and human geography. Transactions of the Institute of British Geographers, Reino Unido, 29, p. 282-295, 2004.

PICKLES, John (ed). Ground Truth: The social implications of geographic information systems. New York, Guilford Press, 1995.

PICKLES, John. Tool or science?: GIS techno-science and the theoretical turn. Annals of the Association of American Geographers, Estados Unidos, 8: p. 363-372, 1997.

PICKLES, John. Arguments, databases and dialogues: the GIS- social theory debate and the concern for alternatives in LONGLEY, Paul, GOODCHILD, Michael F., MAGUIRE, David J., RHIND, David W. (ed.). Geographical Information Systems. Segunda edición. New York, John Wiley \& Sons, 1999.

RAPER, Jonathan y LIVINGSTON, David. Let's get real: spatio-temporal identity and geographic entities. Transactions of the Institute of British Geographers, Reino Unido, 26, 2001.

REYES, Carmen. Cyber-cartography from a modeling perspective. In: TAYLOR, D. R. F. (ed.). Cyber-cartography: theory and practice. Reino Unido, Elsevier Scientific, 2005.

REYES, Carmen y MARTINEZ, Elvia. Technology and culture in cyber-cartography. In: TAYLOR, D. R. F. (ed.). Cyber-cartography: theory and practice. Reino Unido, Elsevier Scientific, 2005.

SHEPPARD, E.S y POIKER, Tom (ed.). Special Issue: GIS and society. Cartography and GIS, Estados Unidos, 22:1, 1995.

SCHUURMAN, Nadine. Trouble in the heartland: GIS and its critics in the 1990s. Cartographica, Canadá, 36,4, 1999.

SCHUURMAN, Nadine. Reconciling social constructivism and realism in GIS. ACME International E Journal for Critical Geographies, Canadá, 1:1, 2002

WRIGHT, Dawn J., GOODCHILD, Michael F. y PROCTOR, James D. GIS tool or science? demystifying the persistent ambiguity of GIS as "tool" versus "science". Annals of the Association of American Geographers, Estados Unidos, 87:2, 1997.

WRIGHT, Dawn J., GOODCHILD, Michael F. y PROCTOR, James D. Reply: still hoping to turn that theoretical corner. Annals of the Association of American Geographers, Estados Unidos, $87: 2,1997$.

SILVANA LEVI - Professora do Centro de investigación en Geografía y Geomátia "Ing. Jorge L. Tamoyo". Doutora pela Universidade Autônoma do México com Pós-doutorado pela universidade Nattinghan-Inglaterra. 